\title{
Model on Analysis of Industrial Relation Based on the Binary Relation Theory
}

\author{
Kai-ya $\mathrm{Wu}^{1}$, Xiao-jian Chen ${ }^{1}$, Jia-zhong Qian ${ }^{2}$, and Ru-zhong $\mathrm{Li}^{2}$ \\ 1 School of Business, University of Science and Technology of China, \\ Hefei, 230026, China \\ wuky2000@vip.sina.com \\ 2 School of Natural Resources and Environmental Engineering, \\ Hefei University of Technology, Hefei, 230009, China \\ qjzy@hfut.edu.cn
}

\begin{abstract}
Based on the binary relation theory and Warshall's algorithm, a model on the connected incidence relation of the industry system is set up, and a handy method of quantitative analysis is provided for understanding the industrial structure and the relation. The model is applied to analyze the connected incidence relation of the industrial system in Anhui Province. The results show the model is effective with simple principle and handy operation. And it provides accurate reference for the analysis of the connected effects among different industries as well as the adjustment and optimism of the industrial structure.
\end{abstract}

\section{Introduction}

The binary relation indicates certain relevance between two elements in the set, which largely exist in the economic phenomenon. There are various relation and affections of different levels in the industrial system. By means of the inputoutput table, the analysis of the industrial relation aim to analyze the relation happening in the process of producing, distributing and exchanging among industries (Zhi-biao Liu, 2001).

In recent years, the analysis methods have been improved a lot. For instance, the optimal theory, as well as the Graph Model (Bing-xin Zhao, 1996.) and Degree-Hierarchy Structure Model (Liu and Zhou, 1999) are used in the analysis of the industrial relation. This essay utilizes exploringly the binary relation theory to establish the incidence relation of the industry system and to calculate the connected incidence relation through Warshall's algorithm. From this, it defines the influential industrial set under analyzing the industrial relation of industry system and the evolving regular of industrial structure.

\section{Principle \& Method Base}

\subsection{Establish the Incidence Relation $r$ among Industries in the Industrial System}

Take the whole industrial system as a set $S$ : take the industries divided according to a certain principle as $1,2,3, \cdots n$-the elements of $S$, then it comes that $S=$ $1,2,3, \cdots n$. Establish the incidence relation $r$ of the set $S$ : 


$$
r=\left\{(i, j) \mid i \in s, j \in s, d_{i, j} \geq \alpha\right\}
$$

In this expression, $\widetilde{d}_{i j}$ is determined by W.leontief's inverse matrix $\widetilde{D}=(\widetilde{d})_{i j}$ and $\alpha$ is the incidence critical value given previously. The compound relation between the incidence relation $r$ and $r$ :

$$
r \circ r=\{(p, q) \mid p \in s, q \in s, \exists t \in s((p, t) \in r,(t, q) \in r)\}
$$

If $(p, q) \mid p \in r \circ r$, that is, an industry $t$ exists in the whole system $S$, the incidence relation $r$ then happens between $p$ and $t$. Meanwhile, anther $r$ happens between $t$ and $q$. therefore, by the compound calculation of the incidence relation $r$, a larger incidence relation can be obtained in the system $S$.

We mark the relational matrix of $r$ as $M_{r}=\left(\alpha_{i j}\right)_{n \times n}$, in which

$$
y= \begin{cases}1 & (i, j) \in r \\ 0 & (i, j) \notin r\end{cases}
$$

We marked the relational matrix of $r \circ r$ as $M_{r \circ r}$. If there's at least one industry $t$ in $S$, which makes a relation between $p$ and $t$, namely $(p, t) \in r$.and a relation between $t$ and $q$, namely $(t, q) \in r$, then a relation between $p$ and $q$ must form $(p, q) \in r$. However, there may not only one industry $t$ that can meet the system $S$, another one $t$ can also satisfy the quest $\left(p, t^{\prime}\right) \in r,\left(t^{\prime}, q\right) \in r$. In all these situation, $(p, q) \in r \circ r$ will be available.

In this way, when we scan row $p$ and column $q$ of $M_{r}$, if we find at least one $t$ which makes the number of $t$ and $q$ is 1 , then the number of the site of row $t$ and column $q$ is also 1 . Otherwise, it will be 0 . Scan one row of $M_{r}$ and each column of $M_{r}$, we will know $M_{r \circ r}$ and all the other rows of $M_{r o r}$ can also be obtained in the similar way.

Thus, we'll get $M_{\text {ror }}$ through Boolean's plus method of matrix. That is,

$$
M_{r \circ r}=M_{r} \circ M_{r}=\left(b_{p q}\right)_{n \times n} \text {, in which } b_{p q}=\vee_{t=1}^{n}\left(\alpha_{p t} \wedge \alpha_{t q}\right) \text {. where, } \vee \text { is Boolean's }
$$

add, conforming to $0 \vee 0=0,0 \vee 1=1,1 \vee 0=1,1 \vee 1=1 ; \wedge$ is Boolean's multiplication, conforming to $0 \wedge 0=0,0 \wedge 1=0,1 \wedge 0=0,1 \wedge 1=1$.

According to the incidence relation $r$ generated from the System $S$, a larger incidence relation $r \circ r$ among the industries of $S$ will be easily got.

\subsection{Establish Connected Incidence Relation}

In the industrial system, the connotation of the relation is abundant while the affections among the industries are complex. From the perspective of inputoutput analysis, W.leontief's anti-coefficient can only reflect the strong and the weak of industrial relation, but not the overall relation.

Besides, any kind of industrial development has double effects. On one side, it increases the supply and improves other industries' developments. On the other side, it stimulates the needs which drive other industries' developments. For this reason, we draw into the connected incidence relation $R$ of the system $S$. 
If $r^{k}=r^{k+1}$ establishes in $r$, then we name $R=r \cup r^{2} \cup r^{3} \cup \cdots \cup r^{k}$ as the connected incidence relation in $S$. In this expression $r^{k}$ indicates that there are $k$ compound calculation in $r$ of $S$, that is $r^{k}=r \circ r \circ \cdots \circ r$ (here, $k$ equals to the number of $r$ ).

Because the industrial system $S=\{1,2,3, \cdots, n\}$ is a limited set, the transitive closure of $r$ is $t(r)=r \cup r^{2} \cup r^{3} \cup \cdots \cup r^{n}$. When $r^{k}=r^{k+1}(k \leq n)$, we have

$$
\begin{aligned}
r^{k+2} & =r^{k+1} \circ r=r^{k} \circ r=r^{k+1}, \\
r^{k+3} & =r^{k+2} \circ r=r^{k+1} \circ r=r^{k} \circ r=r^{k+1}, \\
& \cdots \\
r^{n} & =r^{k+1} .
\end{aligned}
$$

In other words, $r^{k}=r^{k+1}=r^{k+2}=\cdots=r^{n}$. Therefore, the connected incidence relation $R=r \cup r^{2} \cup r^{3} \cup \cdots \cup r^{n}$.

Thus by adopting the famous Warshall's algorithm to calculate the transitive closure, we can quickly get the incidence relational matrix $M_{R}$ of the connected incidence relation $R$.

If matrix $M_{R}=\left(c_{i j}\right)_{m \times n}$ already got through Warshall's algorithm (Kolman, $2001)$, the set $\left\{j \mid c_{i j}=1\right\}$ is called the put industry set of $j$ in the system, while $\left\{j \mid c_{j i}=1\right\}$ is called the push industry set. Till now, a quite clear quantitative result about the industrial relation in $S$ will emerge.

\subsection{Warshall's Algorithm for Computing Transitive Closure}

Warshall's algorithm is an efficient method for computing the transitive closure of a relation, which based on the construction of a sequence of zero-one matrices. These matrices are $w_{0}, w_{1}, \cdots, w_{n}$, where $w_{0}=M_{R}$ is the zero-one matrix of this relation, and $w_{k}=\left[w_{i j}^{[k]}\right]$.It can computes $M_{t(R)}$ by efficiently computing $w_{0}=M_{R}, w_{1}, w_{2}, \cdots, w_{n}=M_{t(R)}$.

LEMMA: Let $w_{k}=\left[w_{i j}^{[k]}\right]$ be the zero-one matrix that has a 1 in its $(i, j) t h$ position if and only if there is a path from $v_{i}$ to $v_{j}$ with interior vertices from the set $\left\{v_{1}, v_{2}, \cdots, v_{k}\right\}$. Then $w_{i j}^{k}=w_{i j}^{[k-1]} \vee\left(w_{i k}^{[k-1]} \wedge w_{k j}^{[k-1]}\right)$, whenever $i, j$ and $k$ are positive integers not exceeding $n$.

The Lemma give us the means efficiently to compute the matrices $w_{k}(k=$ $1,2, \cdots, n)$. We display psendocode for Warshall's algorithm.

Procedure Warshall $\left(M_{R}: n \times n\right.$ zero-one matrix)

begin

$$
\begin{aligned}
& w:=M_{R} \\
& \text { for } \mathrm{k}:=1 \text { to } \mathrm{n} \\
& \text { begin } \\
& \quad \text { for } \mathrm{j}:=1 \text { to } \mathrm{n} \\
& \quad w_{i j}=w_{i j} \vee\left(w_{i k} \wedge w_{k j}\right) \\
& \text { end }
\end{aligned}
$$$$
\text { end. }\left\{w=\left[w_{i j}\right] \text { is } M_{t(R)}\right\}
$$ 
The computational complexity of Warshall's Algorithm can easily be computed in term of bit operations. To find the entry $w_{i j}^{[k]}$ from the entries $w_{i j}^{[k-1]}, w_{i k}^{[k-1]}$ and $w_{k j}^{[k-1]}$ using Lemma above requires two bit operations. To find all $n^{2}$ entries of $w_{k}$ from those of $w_{k-1}$ requires $2 n^{2}$ bit operations. Since warshall's algorithm begins with $w_{0}=M_{R}$ and computes the sequences of $0-1$ matrices $w_{0}, w_{1}, \cdots, w_{n}=M_{t(R)}$, the total number of bit operations is $n \cdot 2 n^{2}=2 n^{3}$.

\section{Analysis of Relation for Anhui Industrial System as a Case}

According to the 40 industrial departments classified in Anhui's Input-output Table in 1997 (Anhui's Input-Output Office,1999) and the complete consuming coefficient table, we choose an appropriate communicative critical value $\alpha$ (here $\alpha=0.1)$ to establish the incidence relational matrix $M_{r}$, and to calculate $M_{R}$ by Warshall operation is below(next page).

Based on the $M_{R}$,We can figure out the connected incidence relation of Industrial system directly in Anhui Province. The main results are attained as followings.

(1) There's a group of connected incidence relation in Anhui's system. $S_{0}=\{2,3,4,5,11,12,14,15,16,24,28,33,35\}$,that is Coal mining and selection industry,Petroleum and natural gas mining industry, Metal and nonmetal mining and selection industry, Crude oil product and coke purification industry, Chemical industry, Metal purification and metal products, machine-building industry, Electricity and Vapour products and supply, Freight transportation and preservation, Financial insurance, Social service\}.

The internal development and variation of this group can bring about the relative changes in other industries and at the same time improve those industries outside this group. For their strong overall functions, these industries can be the motive of economic development, which serves as the pivot to maintain economy's stability, compatibility and its high pace.

(2)The group $S_{0}$ includes resource,energy,service and other basic industries.In developing point of view,these industries must have the privilege be developed,which will pave the way for the continual development of Anhui's economy.

(3)Agriculture (industry code 1) is the basic industry that proposes a particular status in Anhui.On one hand,it can improve the development of food,textile,costume and other groups (code 6,7,8,10,22,29) and meanwhile pull the development of the communicative connected group $S_{0}$,so it fully displays that Anhui is a big and strong agriculture province.

(4) Social service (industry code 35), especially the tourist industry,is very important in Anhui.It can not only improve the development of $S_{0}$, but also all the other industry's developments.

(5)Financial industry (industry code 35) has impact on all the industries including $S_{0}$.It will do good to the whole nation's economy. 


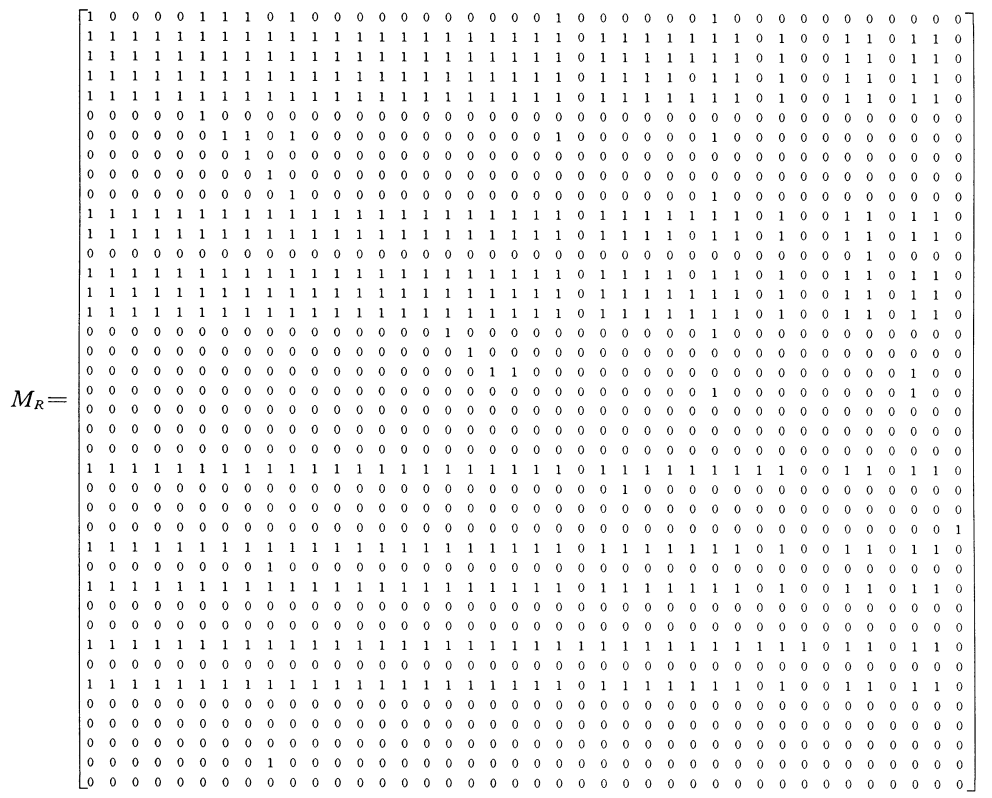

(6)The essence of the dominant industry lies in its leading effect in the industrial structure system.Anhui's practical situation should be taken into account in selecting the dominant industry.But on addition,the agriculture and social service which have special status should also be included.In this way,we can realize the quick increase on the basis of the optimism of the industrial structure.

\section{Conclusion}

From the above-mentioned analysis, conclusions can be reached, the model based on the binary relation theory and Warshall's algorithm is effective with simple sufficient mathematical theories and convenient operation. And the model is applied. By adopting the statistics of the input-output graph, through the computer programming, the connected incidence relation can be obtained quickly under different incidence critical value, which will offer accurate reference for the analysis of the connected effects among different industries as well as the adjustment and optimism of the industrial structure.

Acknowledgements. This research was supported by the National Social Science Foundation of China (02BJY043) and the National Natural Science Foundation of China(40202027). The authors would like to thank the anonymous reviewer for their help. 


\section{References}

1. Zhi-biao Liu:Analysis of Modern Industry Economy.Nanjing University Press (2001)

2. Bing-xin Zhao:Researching on the graph model applied to the industrial relation.Systems Engineering-Theory \& Practice.2 (1997):39-42

3. Yong-qing Liu,Zhou Chan-shi:Degree-hierarchy structure model for the industry system of Guangdong Province and Its application. Systems Engineering -Theory \& Practice.3(1999):116-131

4. Bernard Kolman:Discrete Mathematical Structure.Prentice Hall,Inc(2001)

5. Anhui's Input-Output Office:Anhui's Input-Output Table in 1997 